\title{
Computational aerodynamic analysis of flatback airfoils by coupling N-S equations and transition prediction codes
}

\author{
L. Deng ${ }^{1}$, Y. W. Gao ${ }^{1} \&$ J. T. Xiong ${ }^{2}$ \\ ${ }^{I}$ National Key Laboratory of Science and Technology on Aerodynamic \\ Design and Research, Northwestern Polytechnical University, China \\ ${ }^{2}$ Department of Mechanical and Aerospace Engineering, \\ University of California, USA
}

\begin{abstract}
Flatback (Blunt Trailing Edge) airfoils are adopted for the inboard region of large wind turbine blade due to their structural and aerodynamic performance advantages. Very limited experimental data at high Reynolds Number makes it difficult for wind turbine designers to design and use these section shapes because the wind tunnel experiments are limited by the Reynolds Number and the solid blockage. In this study, a 2-D Reynolds-Average Navier- Stokes Solver coupled with a transition prediction based on the $e^{N}$ method is used to CFD computation of blunt trailing edge airfoils. A new coupling structure with a timeaccurate transition prediction model taking the unsteady flow as a result of the bluff-body vortex shedding into account is developed. The computational grid is $\mathrm{C}$-Grid generated by the tool of Gridgen, and the vertical angle at the blunt trailing edge is smoothed slightly to increase the grid quality. An airfoil of DU97-Flat modified by DU97-W-300 airfoil for wind turbine application is calculated and effects of grid points are investigated. The aerodynamic performance of DU97-W-300 is calculated and comparisons between the results from literature and wind tunnel experimental data are performed, and the results show that the method in present study can obtain the aerodynamics performance with much less grid numbers while agreeing better with the wind tunnel experimental data than the literature. One issue that requires attention is the prediction of maximum lift and the failure to accurately capture stall behaviour by the various computational techniques used in this study.
\end{abstract}

Keywords: wind turbine, airfoil, flatback airfoil, couple, transition prediction. 


\section{Introduction}

In aerodynamic performance prediction and geometry design of horizontal axis wind turbine (HAWT), the airfoil data of lift and drag coefficient for the different airfoils applied along the span play a significant role. As a result, the designer will spend a lot of up-front time to prepare reliable airfoil aerodynamic data. It is believed that the errors in airfoil data tables are the single largest source of error in most rotor load and performance predictions [1-3].

Recently, the blunt trailing edge or called flatback airfoils have been proposed for the inboard region of large wind turbine blades [4-6]. Flatback airfoils provide several structural and aerodynamic performance advantages. Structurally, the flatback increases the sectional area and section moment of inertia for a given airfoil maximum thickness. Aerodynamically, the flatback increases section maximum lift coefficient and lift curve slope and reduces the well-documented sensitivity of the lift characteristics of thick airfoils to surface soiling [7]. But the flow separation and body-off vortex shedding in the flatback region increase the drag also. One of the problems with wind tunnel testing thickness airfoils is that these types of models tend to create a significant amount of solid blockage and wake blockage thereby affecting the measurements and the flow development in the wind tunnel test section. Solid blockage is typically kept at $5 \%$ or less, but this value limits the model chord length, which in turn limits attainable Reynolds Numbers. The Reynolds Numbers are also restricted by load limitations of wind tunnel pyramidal balance. As a result, the published experimental results on flatback airfoils are obtained at low Reynolds Numbers, or are for limited trailing edge bluntness. The lack of experimental data precipitates the analysis of flatback airfoils using computational fluid dynamics (CFD). Recent years, the analysis of flatback airfoils using CFD tend to using more and more grid numbers to capture the vortex structure, but the transition positions are generally obtained by a transition position prediction code and then used as a fixed transition position in the N-S solver and therefore that does not count for the unsteady nature of the flow field because of the blunt trailing edge [8-12]. In present study, several computational techniques are applied including using an N-S solver coupling a transition position prediction code and transition flow region model, and the transition positions are predicted counting the unsteady nature of flow.

\section{Flow solver}

The aerodynamic performance characteristics of flatback airfoils are calculated using CFD code based on Reynolds-average Navier-Stokes (RANS) equations. The code solves the compressible, two-dimensional, RANS equations. The governing equations are central differenced in standard second-order form and second- and fourth-order artificial dissipation terms are added for numerical stability. The code employs local time stepping and implicit residual averaging, multi-grid technique to accelerate convergence. 
In present RANS solver, the one-equation Spalart-Allmaras (S-A) and the zero-equation Baldwin-Lomax (B-L) model are available. Although both models have been successfully applied to study flatback airfoils, the S-A model is selected because the results show that it gives a better agreement with the experimental data in separated flows.

\section{The effects of grid}

To reduce the calculation time, it is reasonable to keep the number of grid points at a minimum. This minimum is defined by the number of grid points, which is necessary to generate accurate results. In order to define the minimum number of grid points, different grid spaces are tested. A flatback airfoil of DU97-flat (see Fig. 1) is selected and that is created by adding thickness to the aft half of the airfoil of DU97-W-300, giving a blunt trailing edge with a width of $10 \%$ chord [10]. The lift coefficient at different grids numbers are calculated at $\operatorname{Re}$ of $3 \times 10^{6}$ and Mach number of 0.165 , angle of attack of $0^{\circ}$, and at that condition the transition position are fixed at 0.343 (upper surface) and 0.320 (lower surface).

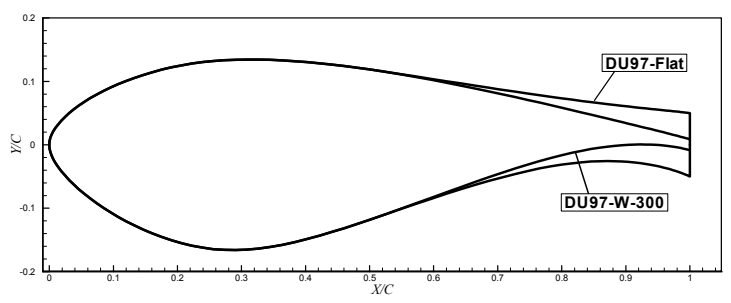

Figure 1: $\quad$ Airfoil shapes of DU97 and DU97-flat.

In previous study, in order to keep cell skewness minimal and the angles in the cells close to $90^{\circ}$, a C-grid works well with sharp trailing edges. When the trailing edge grows thicker, the cell skewness increases and the grid-shocks appear at the blunt base; in that case, the O- grid in contrast does not generate grid shocks and works well with blunt trailing edges [11]. But in the present study, in order to compare with sharp trailing edge, C-grid is generated using the grid generation tool called Gridgen. In grid generating, the vertical angle at the blunt trailing edge is smoothed slightly to increase the grid quality, and that is common for this kind of airfoils [13]. In this study, the RANS equations are solved coupling a transition position prediction code to obtain the pressure distribution of boundary layer. The accuracy of the parameters of the boundary layer influence the accuracy of the transition position prediction, therefore an initial spacing is $1.0 \times 10^{-6}$ and the first 60 points are with equivalent spacing. Table 1 shows a series of grid numbers. Fig. 2 shows the C-grid of $352 \times 96$ that was used and a close-up of the trailing edge. 
48 Computational Methods and Experimental Measurements XV

Table 1: Different computational grid.

\begin{tabular}{ccccc}
\hline Grid & wall & wake & vertical & far field/chord \\
\hline $256 \times 80$ & $96 \times 2$ & 32 & 80 & 12 \\
$352 \times 96$ & $128 \times 2$ & 48 & 96 & 20 \\
$400 \times 104$ & $152 \times 2$ & 48 & 104 & 20 \\
$448 \times 112$ & $160 \times 2$ & 64 & 112 & 20 \\
$496 \times 112$ & $184 \times 2$ & 64 & 112 & 30 \\
$544 \times 128$ & $192 \times 2$ & 80 & 128 & 30 \\
\hline
\end{tabular}
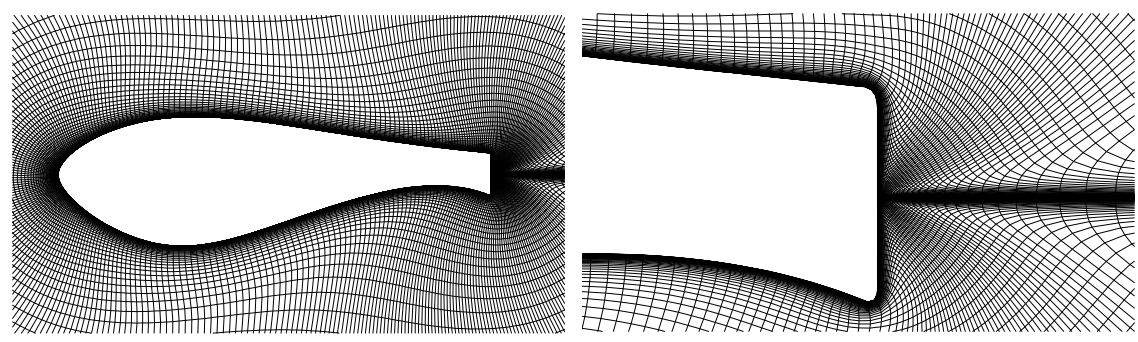

Figure 2: $\quad$ Computational grid of DU97-flat and close up.

Fig. 3 shows the calculated lift coefficient versus the number of grid points. We can see from the figure that with grid number increasing, the lift coefficient tends to converge toward a constant value, and in order to obtain enough accurate aerodynamic performance, we should increase the number of grid points but that brings a increase of computational cost; as a result, we should try to find a trade-off between them. In the following calculation, the number of grid is selected as $448 \times 112$.

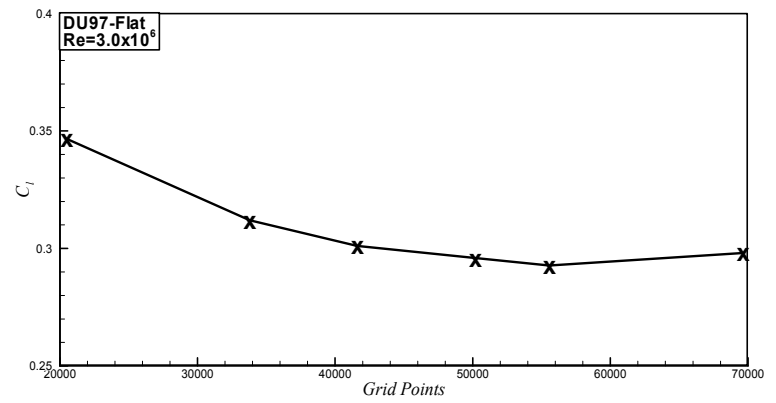

Figure 3: Comparison of Lift coefficient for different number of grid points. 


\section{Transition prediction and transition flow model}

\section{$4.1 e^{N}$ method}

The $e^{N}$ method based on linear stability analysis is used as a transition criterion. For a set of specified dimensional frequencies, the amplification $\mathrm{N}$ factor is computed as:

$$
N=-\int_{x_{0}}^{x} \alpha_{i} d x
$$

here, $x_{0}$ corresponds to the location where amplification rate $\alpha_{i}$ is zero. A group of amplification curves (in present study, 15 curves) for different dimensional frequencies are computed and then to determine the first dimensional frequency that is referred to as the critical frequency which achieves the predetermined amplification factor corresponding to transition.

\subsection{Transition flow model}

In a previous study, usually the point transition is applied in the N-S solver, that is defined by merely switching on the turbulence model at transition onset. On one hand, this procedure results in an eddy viscosity production that yields rapid but not abrupt changes of the viscous layer properties; thus a small transition zone is created computationally. On the other hand, point transition represents a local perturbation due to the relatively sharp reduction in the displacement thickness in the vicinity of transition, which produces a strong viscous/inviscid interaction region with a remarkable upstream influence. Considerable perturbations in wall pressure and in viscous layer properties are present, which prevent the iteratively coupling N-S and $e^{N}$ method computations to converge. To the contrary, the application of a finite length transition zone in N-S computations will be shown to reduce the strength of the local perturbation; correspondingly, the changes in wall pressure and viscous flow data will be more moderate. In present study, we use the Walker's model [14], which is initially defined as:

$$
\operatorname{Re}_{\Delta X}=5.2 \operatorname{Re}_{X^{T}}^{3 / 4}
$$

here, $\Delta X$ is the transition zone length, and $X^{T}$ is the location of transition onset. However, Walker's transition length model is based on restrictive assumptions; such that the estimate is considered represent the minimum length. Walker proposed to use the double value of the minimum length model, and then the model is:

$$
\operatorname{Re}_{\Delta X}=10.4 \mathrm{Re}_{X^{T}}{ }^{3 / 4}
$$

This model is applicable for flow situations where transition is predicted well upstream of laminar separation, but numerical experiments show that in cases small separation bubbles, this model is available as well. 


\section{Coupling of RANS and $e N$ method}

In published literatures on the aerodynamic performance computation of flatback airfoils, the transition locations are computed by a transition prediction code and then use the locations as fixed transition in RANS solver. But because the vortex shedding in the trailing edge, the calculated coefficients show cycle changes, thus the transition location prediction should count the unsteady of the flow field. In this study, the algorithm for the coupling of RANS and transition prediction works as follows:

a) The RANS solver is started with prescribed transition locations on upper and lower sides of the airfoil. In this moment, the locations are usually set very far downstream, such as 0.8 chord or even at the trailing edge (in the latter case, the RANS solver computes a fully laminar flow).

b) The RANS equations are iterated cycle $_{\max }$ times, in that moment the coefficients have come to the stage of cycle change.

c) The computation is continued next $\Delta k c y c$ times, and averaged pressure distribution of wall and boundary layer are computed, and the computed properties are used as inputs in the boundary layer equations solving. And then, the velocity profile and boundary layer parameters such as displacement thickness, momentum thickness and shape factor are computed.

d) The transition prediction module is called to transition onset coordinate of $x^{T}$. From the instability point to downstream, the $e^{N}$ method is used to calculate the amplification factor $\mathrm{N}$ to check whether it is the onset point of transition. At every point, before the calculation of $\mathrm{N}$ factor, a check of whether the laminar separation has occurred; in the case of laminar separation, the separation point is used as an approximation of the transition location.

e) The current coordinate $x^{T}$ is under-relaxed to use as the transition location. A new transition location $x^{*, T}$ is defined as:

$$
x^{*}, T=C^{T} x^{T}
$$

here, $C^{T}>1$. The under-relax of the determined transition location prevents the case that at an unconverged stage, the transition location is determined to far upstream and might not be shifted to downstream again. According to Krumbein, the new location can be defined as ${ }^{[15]}$ :

$$
x_{l}^{*, T}=x_{l-1}^{*, T}-f\left[x_{l-1}^{*, T}-x_{l}^{T}\right]
$$

here, $f$ is 0.7 , and $l$ is current iteration step of transition prediction.

f) As convergence criterion, $\Delta x_{l}^{*, T}<\varepsilon=.01$, with $\Delta x_{l}^{*, T}=\left|x_{l}^{*, T}-x_{l-1}^{*},\right|$. In the case that criterion is satisfied, the iteration is finished; else the algorithm loops back to the station of $\mathrm{c}$ ) using the transition location of $x_{l}^{*}, T$. 
We use the DU97-flat airfoil as a validation at Re of $3 \times 10^{6}$ and Mach number of 0.165 , angle of attack of $0^{\circ}$. The initial transition locations are set at 0.8 at both sides with cycle $_{\max }$ of 1500 and $\Delta k c y c$ of 200 . Fig. 4 shows the curves of the transition locations versus iteration steps of transition location, and it shows that within 10 steps the transition locations converge on constant values. Fig. 5 shows that the lift and drag coefficient converge toward their converged values. From this case we can see that the coupling method in present study can be used in the computing of the aerodynamic performance of flatback airfoils.

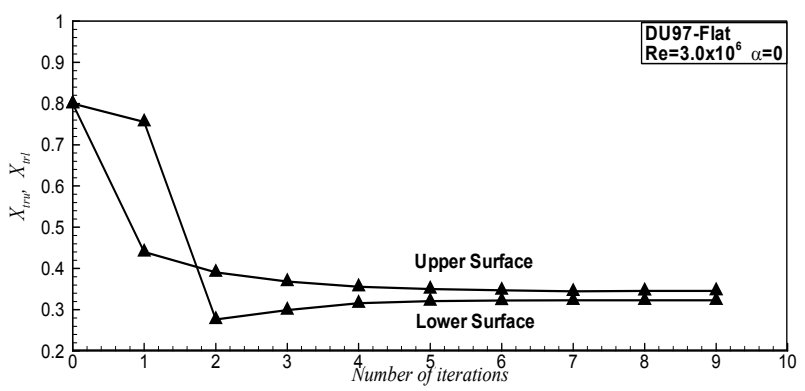

Figure 4: Convergence history of the transition locations.

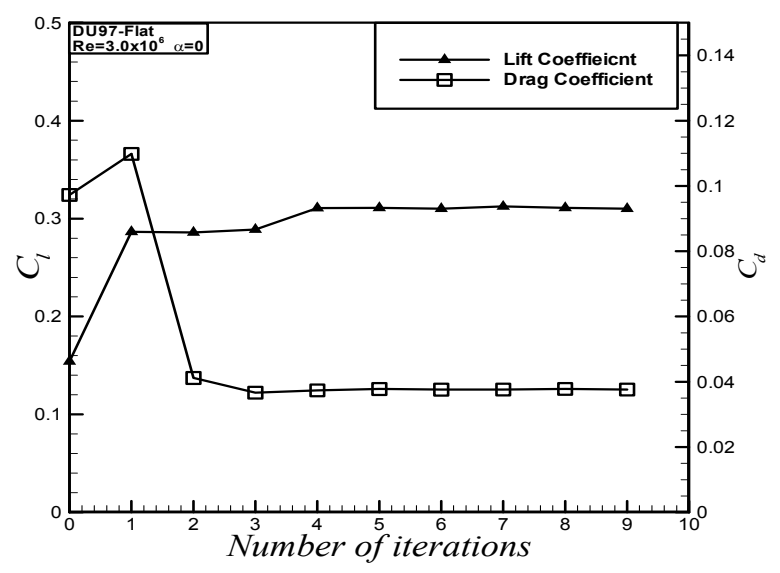

Figure 5: Convergence history of lift and drag coefficient.

\section{Results and discussion}

In 1999, Timmer published a wind tunnel measured data of airfoil of DU97-W300 at Reynolds number of $3.0 \times 10^{6}$ and Mach number of 0.165 [16]. The DU97$\mathrm{W}-300$ is a wind turbine airfoil with a maximum thickness of $30 \%$ chord and a trailing edge thickness of $1.74 \%$ chord. Matthew computed DU97-W-300 with a RANS solver of SACCARA and used almost $4 \times 10^{5}$ grid points, but the transition 
locations were computed by the code of Xfoil and then used as fixed locations [10]. In the present study, we compute DU97-W-300 and compare the results with experimental data and Matthew's results. Fig. 6 is the comparison of transition locations computed by the coupling method in this paper and Xfoil code. We can see that at low angle of attack, the transition locations at lower surface agrees well with Xfoil results, but with some discrepancies appearing with angle of attack growing higher. The computed results in present study are smaller than Xfoil results; especially at angle of attack of 12 degree, the computed results show a separation at leading edge, but there is still 0.1 chord laminar flow in Xfoil results.

Fig. 7 compares the lift coefficient and moment coefficient. A better agreement is shown in lift coefficient between the computed and experimental lift results than the SACCARA results, especially in linear region; but just as Matthew's results show, the computed results did not capture the stall angle. And

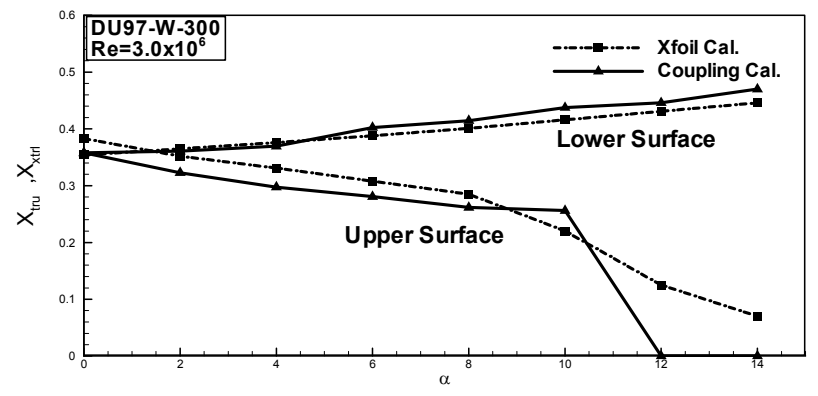

Figure 6: Comparison of transition locations.
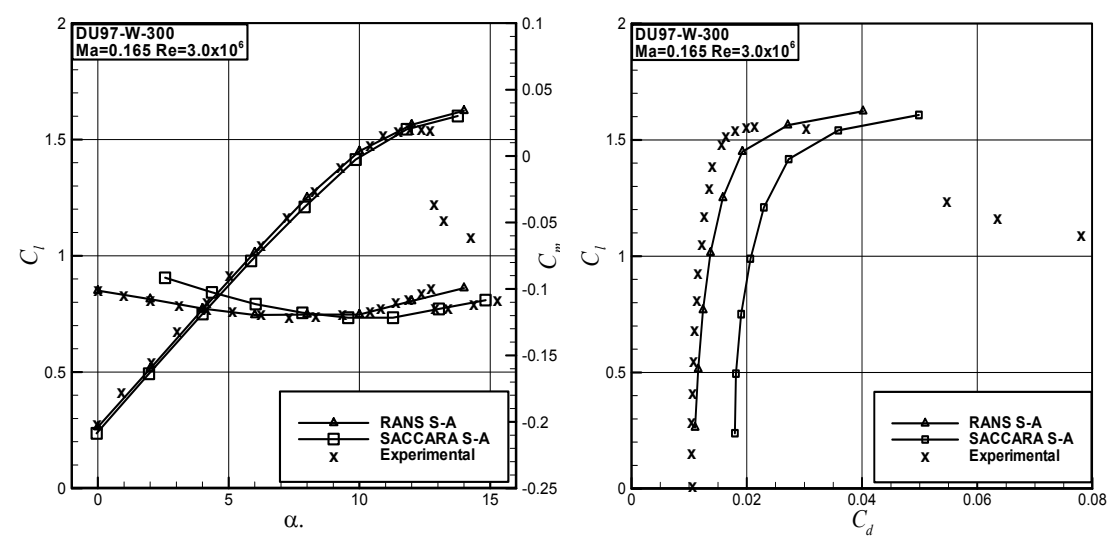

Figure 7: Comparison of lift and moment coefficient comparison of drag polar. 
very good agreement is shown in moment coefficient before angle of attack 13 degree. Fig. 8 is the comparison of drag coefficients. The SACCARA' results are almost double of experimental data and the computed results show a good agreement with the experimental data but there are some discrepancies at high lift coefficient. And it is interesting that there is a constant difference between the SACCARA' results and the computed results in this study.

\section{Conclusion}

Flatback airfoils are considered for the inner regions of large wind turbine blades. The concept of blunt trailing edge is nothing new and has been previous investigated by CFD method. However, previous studies are typically based on a RANS solver using a fixed transition locations computed from a transition prediction code, which don't take the unsteady nature of flow field because of the vortex shedding at the trailing edge into account. From the investigation of the effects of grid points, a trade-off should be made between the growth of the number of grid points and the computational time. A RANS solver is coupled with a transition prediction code with a time-accurate transition model. The results show that the transition locations and the computed aerodynamic coefficient converge toward constant values within 10 iteration steps. The wind turbine airfoil of DU97-W-300 is computed by the coupling method and the results are compared with experimental results and the results from the literature. The comparisons show that the computed results agree better with the experimental data than the results in the literature while with less grid points. One issue that requires attention is the prediction of maximum lift and the failure to accurately capture stall behavior by the various computational techniques used in this study.

\section{References}

[1] Patrick J. AeroDyn Theory Manual, NREL/EL-500-36881, 2005.

[2] Simms D., Schreck S., Hand M., Fingersh L.J. NREL Unsteady Aerodynamics Experiment in the NASA-Ames Wind Tunnel: A Comparison of Predictions to Measurements. NREL/TP -500-29494, 2001.

[3] Tangler, J.L. The Nebulous Art of Using Wind-Tunnel Airfoil Data for Predicting Rotor Performance. NREL/CP-500-31243, 2002.

[4] TPI Composites, Innovative Design Approaches for Large Wind Turbine Blades - Final Report, SAND2004-0074, 2004

[5] Standish, K.J., and van Dam, C.P., Aerodynamic Analysis of Blunt Trailing Edge Airfoils, Journal of Solar Energy Engineering, 125(4), pp. 479-487, Nov. 2003

[6] Jackson K, Zuteck, M., van Dam, C.P., Standish, K.J., and Berry, D., Innovative Design Approaches for Large Wind Turbine Blades, Wind Energy, 8(2), pp. 141-171, 2005 
[7] Van Rooij R.P.J.O.M., and Timmer W.A., Roughness Sensitivity Considerations for Thick Rotor Blade Airfoils, Journal of Solar Energy Engineering, 125(4), pp. 468-478, Nov. 2003

[8] Baker J., Mayda E., and van Dam C.P. Computational and experimental analysis of thick fatback wind turbine airfoils, AIAA Paper 2006-193, 2006.

[9] D.D. Chao, and C.P. van Dam, RaNS Analysis of an Inboard Flatback Modification on the NREL Phase VI Rotor, AIAA Paper 2006-195,2006

[10] Matthew F. Barone, Dale Berg. Aerodynamic and Aeroacoustic Properties of a Flatback Airfoil: An Update, AIAA Paper 2009-271, 2009

[11] TPI Composites. Computational Design and Analysis of Flatback Airfoil Wind Tunnel Experiment, SAND 2008-1782, 2008

[12] Christopher Stone, Matthew Barone, C. Eric Lynch, et al. A Computational Study of the Aerodynamics and Aeroacoustics of a Flatback Airfoil Using Hybrid RANS-LES, AIAA 2009-273, 2009

[13] T. Winnemöller, Design and numerical optimization of thick airfoils, AIAA 2006-238, 2006

[14] G.J. Walker, Transitional Flow on Axial Turbomachine Blading, AIAA Journal, 27(3), pp. 595-602, 1989

[15] A. Krumbein. Navier-Stokes Airfoil Computation with Automatic Transition Prediction Using the DLR TAU Code- A Sensitivity study, New Results in Numerical and experimental Fluid mechanics V., Springer Berlin/ Heidelberg, Vol. 92, 2006

[16] Timmer, W.A., and van Rooij, R.P.J.O.M. Design and Wind Tunnel Test of Airfoil DU 97-W-300, IW-98003R, Institute for Wind Energy, Delft University of Technology, 1999 